\title{
Temperature and Field Dependence of the Mobility in Liquid-Crystalline Conjugated Polymer Films
}

\author{
S. J. Martin and A. B. Walker \\ Department of Physics, University of Bath, Claverton Down, Bath BA2 7AY, UK \\ A. Kambili \\ Department of Physics, University of Bath, Claverton Down, Bath BA2 7AY, UK and \\ Institut fuer Theoretische Physik, Universitaet Regensburg, \\ Universitaetsstrasse 31, Regensburg D-93053, Germany
}

\begin{abstract}
The transport properties of organic light-emitting diodes in which the emissive layer is composed of conjugated polymers in the liquid-crystalline phase have been investigated. We have performed simulations of the current transient response to an illumination pulse via the Monte Carlo approach, and from the transit times we have extracted the mobility of the charge carriers as a function of both the electric field and the temperature. The transport properties of such films are different from their disordered counterparts, with charge carrier mobilities exhibiting only a weak dependence on both the electric field and temperature. We show that for spatially ordered polymer films, this weak dependence arises for thermal energy being comparable to the energetic disorder, due to the combined effect of the electrostatic and thermal energies. The inclusion of spatial disorder, on the other hand, does not alter the qualitative behaviour of the mobility, but results in decreasing its absolute value.

PACS: 72.10.-d, 72.80.Le
\end{abstract}

\section{INTRODUCTION}

Conjugated polymers, which derive their semiconducting properfies from delocalized $\pi$ bonding along the polymer chaind, enable cheap, lightweight, portable and large area displays, with mromising applications to solar cells

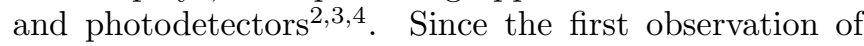
polymer light-emitting diodes (LEDs) tigation of the aspects of chemistry, physics and engineering of materials has resulted in rapid progress 6 . In order to commercialize polymer devices, high efficiencies, brightness, and carrier lifetimes are required $\mathrm{f}$. It is, therefore, essential to fully understand the fundamental physics of electrical transport through conjugated polymers, for a recent review seel.

Previous theoretical studies have dealt with strongly disordered organic materials, in which charge transport is mainly attributed to hopping 10.11 . The charge carrier mobilities of such systems are low and exhibit a strong temperature and electric field dependence. However, large carrier mobilities are generally highly desirable Most recently, measurements in light-emitting diodes $12,13.44$ have demonstrated enhanced carrier mobilities, varying only weakly with the electric field, for devices characterized by high degree of order in the polymeric material. This weak dependence, which contradicts the theoretical predictions, has been attributed to the purity of the polymer films, but it is not yet understood.

This realization has motivated us to investigate the transport properties of liquid-crystalline conjugated polymer films in which the chains are nematically aligned perpendicular to the direction of transport. In a previous work 15 we looked into the character of transport through such polymer films. By employing the Monte
Carlo technique, we showed that it is possible to obtain non-dispersive transport in such systems. This observation is in agreement with time-of-flight experiments conducted on liquid-crystalline polyfluorene films 2 . 13 , which demonstrate non-dispersive hole transport with enhanced charge carrier mobilities compared to previously examined conjugated polymers. Moreover, we established the conditions under which such transport is retained. However, our initial model was a simplistic one, with the film morphology reduced to occupied sites of a twodimensional lattice, the variation of the hopping probability with the field being rather crude, and there was no temperature dependence. In order to accomplish more detailed studies, we have first extended the model to fully describe the geometry of the polymer film, so that various film morphologies, similar to those appearing in realistic systems, can be considered. Secondly, we have explicitly included all parameters of interest, such as the electric field, temperature, and disorder (both spatial and energetic).

The aim of the present work is to probe in detail the field and temperature dependence of the mobility in liquid-crystalline conjugated polymer films. In particular, we discuss the effect of the electric field on the interchain mobility of such polymer films, and we attempt to explain the weak dependence on the field. The interplay between electric field and temperature on the transport characteristics is also examined. We begin our investigation with spatially ordered films in which all polymer chains are perfectly aligned perpendicular to the direction of the field. The additional effect of spatial disorder in the film configurations on the transport properties of such films, is also considered. To account for the chemical regularity and the extended backbone conjugation of 
liquid-crystalline chains we have included only a small amount of energetic disorder.

\section{THE MODEL}

In order to investigate the transport properties of charge carriers moving within a liquid-crystalline polymer film, we have performed numerical simulations of the time-of-flight technique. The system under study contains a polymer film sandwiched between two electrodes, and charge carriers are generated on one side by illumination of the electrode with an intense pulse of light of short duration. The photogenerated carriers move within the bulk of the film under the effect of an external bias, whose sign determines the type of the charge carriers that will get transported.

The film is composed of conjugated polymer chains of length $L=100 \mathrm{~nm}$, which are nematically aligned perpendicular to the direction of the electric field, chosen as the $x$ direction. For the construction of the film periodic boundary conditions have been applied along the other two directions. Based on the extended backbone conjugation of liquid-crystalline polymers, such as polyfluorene, that makes them stiff, and on bond vibrations being of very high frequency and low amplitude, we have assumed that the polymer chains can be described as rigid rods. The thickness of the film in the direction of transport has been taken equal to $d=1 \mu \mathrm{m}$, since relatively thick films are required for such measurements.

Hopping motion of the charge carriers under the influence of the electric field is assumed, which in general can be either intra- or inter-chain. This is described by the Monte Carlo technique, in which the carriers perform random walks within the bulk of the film until they reach the collecting electrode. The probability of hopping between two sites $i$ and $j$ is equal to

$$
\mathcal{P}_{i j}=p_{i j} / \sum_{i \neq j} p_{i j}
$$

where $p_{i j}$ is the unnormalized hopping probability, which is taken to be of the form

$$
p_{i j}=\gamma \exp \left(-\frac{\varepsilon_{j}-\varepsilon_{i}-e \mathbf{E} \cdot \mathbf{r}_{\mathbf{i j}}}{2 K_{B} T}\right)
$$

$\mathbf{E}$ is the electric field, $\mathbf{r}_{\mathbf{i j}}$ is the relative position vector, $K_{B}$ is the Boltzmann factor, $T$ is the temperature, and $\gamma$ denotes the electronic wavefunction overlap. Here, we have considered only nearest-neighbouring hopping within a cutoff distance equal to $10 \AA . \varepsilon_{i}$ is the on-site energy of site $i$ and is taken from a Gaussian distribution

$$
\rho(\varepsilon)=\frac{1}{\sqrt{2 \pi \sigma^{2}}} \exp \left(-\varepsilon^{2} / 2 \sigma^{2}\right)
$$

whose width $\sigma$ determines the degree of energetic disorder present in the film. Notice that in Equation 2 there is no other activation energy except the difference in the electronic site energies a carrier has to overcome during each jump. Equation 2 satisfies the principle of detailed balance 20 .

Intra-chain charge motion is, in general a very rapid process compared with the inter-chain one 16 . Moreover, it was recently shown that in liquid-crystalline polymer films inter-chain hopping accounts for non-dispersive transport 15. Taking these into account, we have mainly focused on inter-chain transport, so that in what follows, each Monte Carlo step corresponds to the time required for an inter-chain jump.

The drift of the photogenerated carriers under the external biass results in a time-dependent current $I(t)$, which reads 17

$$
I(t)=\frac{\partial}{\partial t} \int_{0}^{d} \mathrm{~d} x \rho(x)\left(\frac{x}{d}-1\right)
$$

$d$ is the film thickness, and $\rho(x)$ is the charge density in the direction of transport, integrated over the $y$ and $z$ directions. From the current transient the transit time $t_{T}$, which is the time the carriers need to reach the discharging electrode, is obtained. We then use this extracted transit time to get the mobility $\mu$ via the relation

$$
\mu=\frac{d}{E t_{T}}
$$

\section{RESULTS AND DISCUSSION}

We present results for the charge carrier mobilities of liquid-crystalline conjugated polymer films, obtained by using the Monte Carlo model described in the previous section. Unless stated otherwise, all inter-chain distances are equal to $10 \AA$. The output of such calculations is the current transient, Eq. A, which arises from the application of the external bias. In Fig. 1 we present a current transient from our numerical simulations for electric field $E=3 \times 10^{5} \mathrm{~V} / \mathrm{cm}$ at room temperature, and for disorder $\alpha=2$, where $\alpha$ is defined as $\alpha=\sigma / K_{B} T_{0}$ and $T_{0}=300 \mathrm{~K}$. The calculated current exhibits the typical behaviour of a time-of-flight signal, with a distinctive plateau followed by a decaying tail, thus shows nondispersive transport 18 . From the current plot we obtain the transit time $t_{T}$, which is required for the calculation of the mobility, by using the current integration mode. The latter gives the transit time as the point at which the current has fallen to half its value in the plateau region. In Fig. 11 the transit time is indicated by the arrow.

In most experimental time-of-flight signals, the current transients initially exhibit a spike, which rapidly falls into the plateau. However, in our results such a pronounced peak is missing 19. We attribute the lack of the initial 
spike to the low degree of disorder present in our system, and in particular near the injecting electrode. A demonstration of the effect of purity, in the initial peak is presented in Fig. 2. Curve (a) shows the current transient for a film with no energetic disorder at all $(\delta \varepsilon=0)$, whereas curve (b) corresponds to the case of $\alpha=2$. Curve (a) has been shifted along the $y$ axis for clarity. After the illumination of the electrode, the simulated photogenerated charge packet begins its motion within the film. If no disorder is present, the packet is adequately described by a delta function moving within the bulk of the film with constant mean velocity. If some disorder is introduced into the system, the propagating packet will become more extended (Gaussian), and the carriers will no longer move together. The resulting change in the charge profile produces a spike in the current transient, as shown in curve (b), even though the disorder that we have considered is too small to give a pronounced peak. Additionally, spatial irregularities near the injecting electrode would lead to much larger peaks 5 , similar to those observed in experiments.

By identifying the transit times from the current transients for various values of the external electric field and for different temperatures, we have calculated the mobility by using Equation 5. Figure 3 shows one set of such results for $\alpha=1$. In this graph the logarithm of the mobility, $\ln (\mu)$, has been plotted as a function of $\sqrt{E}$ for temperatures in the range of $100 \mathrm{~K}$ to $350 \mathrm{~K}$. In this Figure we see that for low temperatures $\ln \mu$ shows a more pronounced dependence on $\sqrt{E}$, with a slight increase at low fields, which switches to a decrease at larger values of the bias. As the temperature increases, and, thus, the thermal energy becomes comparable to the energetic disorder, this variation disappears and the mobility changes only weakly with the field. The reduction of the mobility at large electric fields and at all temperatures is the outcome of the saturation of the transit time, since the electrostatic energy is sufficient for the charge carriers to move fast enough and exit the film at the minimum number of time steps required. However, we should point out that we are not aware of any experimental results which show such a decreasing mobility. This indicates that in reality there are not liquid-crystalline polymer films of such energetic purity, even if they are characterized by perfect alignment of the chains.

In Figure the logarithm of the mobility is shown as a function of $\sqrt{E}$ and for the same range of temperatures as previously, but for disorder $\alpha=2$. In contrast to Fig. 3 , $\ln \mu$ now exhibits a positive gradient at all fields. For small values of $T$ the thermal energy is not adequate to enable the carriers to surmount the energy barriers, and the electrostatic energy becomes important. $\ln \mu$ increases with the field as the electrostatic energy permits the carriers to follow shorter paths within the film. Nevertheless, for larger temperatures, the energetic disorder is rather small, and the thermal and electrostatic energies have similar contributions. Thus, the strong field dependence seen in more disordered systems is smoothed out and the carriers move with a constant velocity. A direct comparison with experimental data 12 shows that our calculations for the mobility at room temperature and for $\alpha=2$ lead to the same qualitative behaviour.

Figure 5 shows the behaviour of the mobility as a function of temperature. The circles correspond to $\alpha=1$ and the squares to $\alpha=2$. In the former case, $\ln \mu$ decreases with $T$ for both small (filled circles) and large (open circles) electric fields. Energetic disorder is relatively small so that the thermal energy dominates, making the charge carriers to remain longer within the film, following larger paths. Hence, the mobility decreases with temperature. This is analogous to carriers being scattered by phonons in conventional semiconductors. When $\alpha$ increases, on the other hand, we see that at a low electric field the mobility increases with temperature (filled squares), whereas for a larger field $\ln \mu$ decreases with $T$ (open squares). For small fields, thermal activation is the leading mechanism for the carriers to overcome the energy barriers. Nevertheless, the energetic disorder is still weak and at large fields longer paths due to the excess thermal energy prevail, as before. This is an important result because it shows how much the mobility is affected by the interplay between electrostatic and thermal energy in the case of small energetic disorder. A similar behaviour of the mobility as a function of temperature has also been observed in other types of polymers within a Master equation approach 20 , although in that case the crossover appears to occur at the same field for all temperatures considered. Moreover, at these small amounts of energetic disorder changes in $\ln \mu$ with temperature are also weak.

Nevertheless, this crossover from increasing to decreasing mobility with temperature is not observed if we depart from the equidistant chains arrangement. In Fig. 6 we show $\ln \mu$ as a function of $\sqrt{E}$ and for various temperatures, for a film in which the liquid-crystalline chains are still all nematically aligned, but at inter-chain distances that vary randomly between $7 \AA$ and $13 \AA$ (irregularly spaced chains). The disorder strength is $\alpha=2$, as in Figure 4 A line has been fitted to the simulation data points to guide the eye. Hopping is only allowed between nearest-neighbours within the same cutoff distance as before $(10 \AA)$, discussed in the previous section. When the chains are irregularly spaced within the film (Fig. 6) the mobility is enhanced with temperature for all values of the electric field, as was shown in recent measurements of polyfluorene film 21. This behayiour resembles that of strongly disordered polymer films $\mathrm{l}$. However, in our case the increase of the mobility with temperature arises from spatial irregularities in the arrangement of the chains within the film. The distribution in the relative position vector leads to variations in the electrostatic energy (Eq. 2), so that thermal activation dominates, and the field dependence of $\ln \mu$ remains weak for all temperatures.

Comparison between Figures 4 and 6 also shows that, even though in both regularly and irregularly spaced 
polymer films $\ln \mu$ varies only slightly with the electric field, the introduction of some spatial disorder leads to the reduction of $\ln \mu$. In the latter case, the charge carriers require more time to reach the discharging electrode as some nearest-neighbouring chains appear in distances larger than $10 \AA$. Such jumps are prohibited, and the carriers must find alternative paths that might be longer. This would lead to greater transit times and, therefore, to smaller mobilities, as seen from Eq. 5 .

When the spatial disorder is retained and the energetic disorder is increased, $\ln \mu$ decreases even more. Moreover, its qualitative behaviour approaches the disordered limit, with a stronger dependence of $\ln \mu$ on the electric field. This is shown in Figure 7, in which $\ln \mu$ is plotted as a function of $\sqrt{E}$ and at room temperature for an irregularly spaced film, for two different strengths of energetic disorder. The diamonds correspond to $\alpha=2$, and the circles to $\alpha=3$. The lines are drawn as a guide to the eye. Not only are the charge carriers obliged to avoid large inter-chain distances, but also they have to surmount larger energy barriers. In this case, the charge carriers rely mostly on the electrostatic energy as a source for the energy required to overcome the barriers, similarly to previously examined disordered films 6 .

From the above discussion it is understood that the weak field and temperature dependence of $\ln \mu$ in liquidcrystalline conjugated polymer films originates from the low energetic disorder present in the film. The addition of spatial disorder, by irregularly spacing the liquidcrystalline chains, does not affect this dependence, but only results in the reduction of the absolute value of the mobility. Nonetheless, it is possible that another form of spatial disorder might be present in such films, and this is the existence of chains which deviate from perfect nematic alignment. In other words, it is possible that during the formation of such polymer films, some chains might appear at an angle $\theta$ with respect to the axis of alignment. We have examined this case by assigning in our simulations a random value of this angle $\theta$ to each chain. The chains were also positioned in random interchain distances, as in Figures 6 and 7 .

Figure 8 shows the outcome of such calculations, in which $\theta$ varies randomly between $0^{\circ}$ and $5^{\circ}$. The logarithm of the mobility is plotted as a function of $\sqrt{E}$, for various temperatures, as before, with energetic disorder $\alpha=2$. The lines are drawn as a guide to the eye. This Figure shows that $\ln \mu$ is only weakly dependent on the electric field, and increases with temperature, in a similar fashion to the case of perfect alignment. Hence, the inclusion of an extra form of spatial disorder does not seem to influence the qualitative behaviour of the mobility, and stresses the importance of low energetic disorder as the factor that determines the degree of dependence on the field and the temperature. Unfortunately, we cannot make any comparisons as to whether the mobility increases or decreases with respect to the perfect alignment case, since our simulations for the randomly oriented case have been performed for films with thick- ness $d=0.5 \mu \mathrm{m}$. Although such film thickness might be considered small for real time-of-flight experiments, we believe that our calculations are able to represent the qualitative behaviour of the mobility for films which show deviations from perfect alignment.

\section{SUMMARY AND CONCLUSIONS}

Liquid-crystalline polymer films are generally expected to be characterized by spatial purity and chemical regularity. In this paper, we have presented calculations of the inter-chain mobility of charge carriers through such polymer films, by employing the Monte Carlo technique. In particular, we have investigated the effect of low energetic disorder in the electric field and temperature dependence of the mobility, in order to account for the weak dependence observed in experiments. Initially, films with no spatial disorder were examined. Our numerical simulations have shown that for relatively clean systems, $\alpha=1$, the mobility decreases with the field, as a result of the saturation of the transit time by the electrostatic energy. Nevertheless, this behaviour does not depict a realistic situation and a higher amount of disorder, $\alpha=2$, was required 5 achieve qualitative agreement with experiments $12,13,21$. When the thermal energy is not enough for the charge carriers to overcome the energy barriers, the electrostatic energy becomes important and $\ln \mu$ increases with the field. The weak field dependence of the mobility arises from equivalent contributions from the thermal and the electrostatic energy when the thermal energy is comparable to the energetic disorder.

The interplay between energetic disorder and thermal energy also appears in the temperature dependence of the mobility at a given electric field. For low energetic disorder, $\alpha=1$, the mobility decreases with temperature at all fields, as is commonly seen in crystalline conductors. For larger energetic disorder, $\alpha=2$, and for constant inter-chain distances, the mobility increases with temperature for small fields, but a crossover to the opposite behaviour appears at larger fields. This result is another demonstration of the combined effect of the electric field and the temperature on the mobility of charge carriers through spatially ordered liquid-crystalline polymer films with low energetic disorder.

When spatial disorder is taken into account, in the form of randomly varying inter-chain distances, the observed crossover from increasing to decreasing mobility with temperature is no longer present. Instead, the mobility alyys increases with $T$, as was seen in the experiments21. Because of the presence of a distribution of the relative position vectors, the effect of temperature is now enhanced, even though the mobility is still only weakly dependent on the electric field. Similar behaviour was also observed in films in which the chains deviate slightly from perfect nematic alignment perpendicular to the direction of the electric field.

Finally, our numerical simulations in liquid-crystalline 
polymer films have helped us to distinguish between the effects of energetic and spatial disorder in such systems. Low energetic disorder, thus, high chemical purity of the chains, is responsible for the weak dependence of the mobility in the field. The inclusion of spatial disorder, associated with irregularities in the arrangement of the polymer chains within the film, does not make this dependence any stronger so long as the energetic disorder remains low. The influence of spatial disorder appears at the quantitative behaviour of the mobility, as it results in decreasing considerably the absolute value of $\ln \mu$ for a given range of fields and at a given temperature. We should note, however, that our model does not allow the calculation of the exact value of the mobility, or make predictions about how many orders of magnitude the mobility might increase or decrease.

We thank Prof A. M. Stoneham for his useful comments and discussions. The authors acknowledge the Engineering and Physical Sciences Research Council (EPSRC), and Sharp Labs of Europe for funding the project. One of the authors, A. K., would also like to thank the Alexander von Humboldt foundation.
* Correspondence address email address: agapi.kambili@physik.uni-regensburg.de

1 M. Pope and C. E. Swenberg, Electronic Processes in Organic Crystals and Polymers, Oxford University Press (1999)

2 P. Gattinger, H. Rengel, D. Neher, J. Appl. Phys. 84, 3731 (1998)

3 P. Peumans, V. Bulović, S. R. Forrest, Appl. Phys. Lett. 76, 2650 (2000)

${ }^{4}$ F. Zhang, M. Svensson, M. R. Andersson, M. Maggini, S. Bucella, E. Menna, O. Inganäs, Adv. Mater. 13, 1871 (2001)

5 J. H. Burroughes, D. D. C. Bradley, A. R. Brown, R. N. Marks, K. Mackay, R. H. Friend, P. L. Burn, A. B. Holmes, Nature 347, 359 (1990)

${ }^{6}$ G. Hadziioannou and P. F. van Hutten, Semiconducting Polymers, Chemistry, Physics and Engineering, WileyVch (2000)

7 R. H. Friend, R. W. Gymer, A. B. Holmes, J. H. Burroughes, R. N. Marks, C. Taliani, D. D. C. Bradley, D. A. Dos Santos, J. L. Brédas, M. Lögdlund, W. R. Salaneck, Nature 397, 121 (1999)

8 A. B. Walker, A. Kambili, S. J. Martin, to be published in J. Phys.: Condens. Matt. October (2002)

${ }^{9}$ H. Bässler, Phys. Status Solidi, 175, 15 (1993)
10 S. V. Novikov, D. H. Dunlap, V. M. Kenkre, P. E. Parris, A. V. Vannikov, Phys. Rev. Lett. 81, 4472 (1998)

11 S. V. Rakhmanova, E. M. Conwell, Synth. Met. 116, 389 (2001)

12 M. Redecker, D. D. C. Bradley, M. Inbasekaran, E. P. Woo, Appl. Phys. Lett. 73, 1565 (1998)

13 M. Redecker, D. D. C. Bradley, M. Inbasekaran, E. P. Woo, Appl. Phys. Lett. 74, 1400 (1999)

14 H. C. F. Martens, O. Hilt, H. B. Brom, P. W. M. Blom, J. N. Huiberts, Phys. Rev. Lett. 87, 086601 (2001)

15 A. Kambili, A. B. Walker, Phys. Rev. B 63, 012201 (2001)

16 A. M. Stoneham, M. M. D. Ramos, J. Phys.: Condens. Matt. 13, 2411 (2001)

17 G. F. L. Ferreira, Phys. Rev. B 16, 4719 (1976)

18 J. C. Scott, B. A. Jones, L. T. Pautmeier, Mol. Cryst. Liq. 253, 183 (1994)

19 H. Scher, E. W. Montroll, Phys. Rev. B 12, 2455 (1975)

20 Z. G. Yu, D. L. Smith, A. Saxena, R. L. Martin, A. R. Bishop, Phys. Rev. B 63, 085202 (2001)

21 D. Poplavskyy, T. Kreouzis, A. J. Campbell, J. Nelson, D. D. C. Bradley, Paper P1.4, Materials Research Society 2002 Spring Meeting, MRS Proceedings 725, Editors: G. E. Jabbour, N. S. Sariciftci, S. T. Lee, S. Carter, J. Kido (2002) 


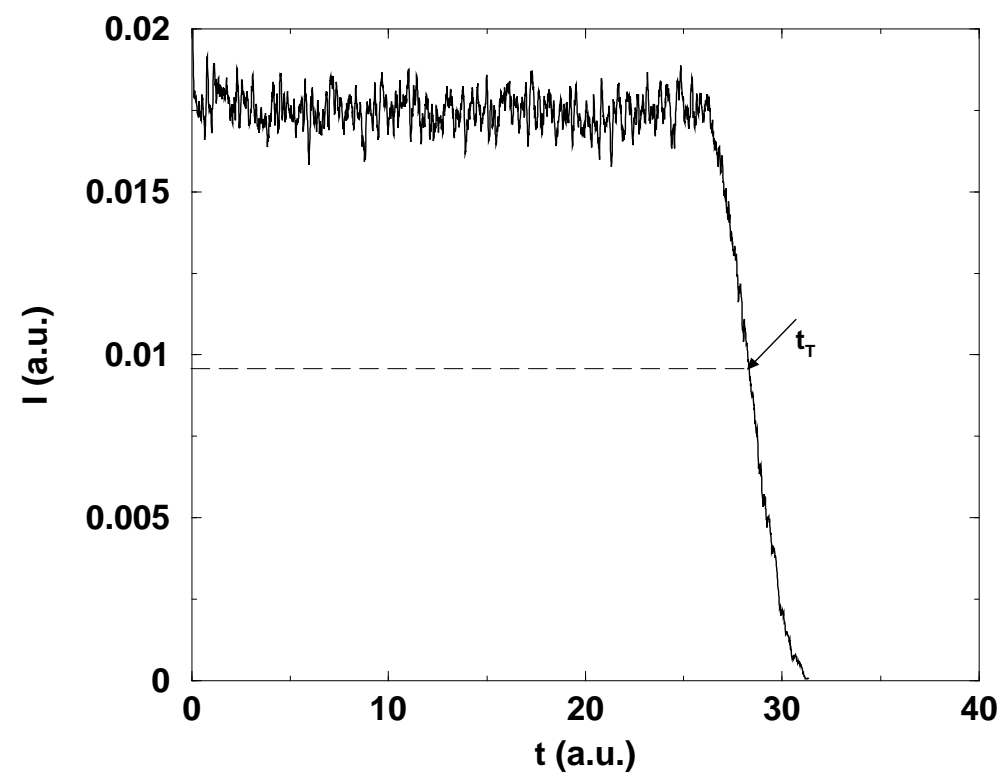

FIG. 1: Current transient for energetic disorder $\alpha=2$, with $E=3 \times 10^{5} \mathrm{~V} / \mathrm{cm}$, and $T=300 \mathrm{~K}$. The transit time $t_{T}$ is indicated by the arrow.

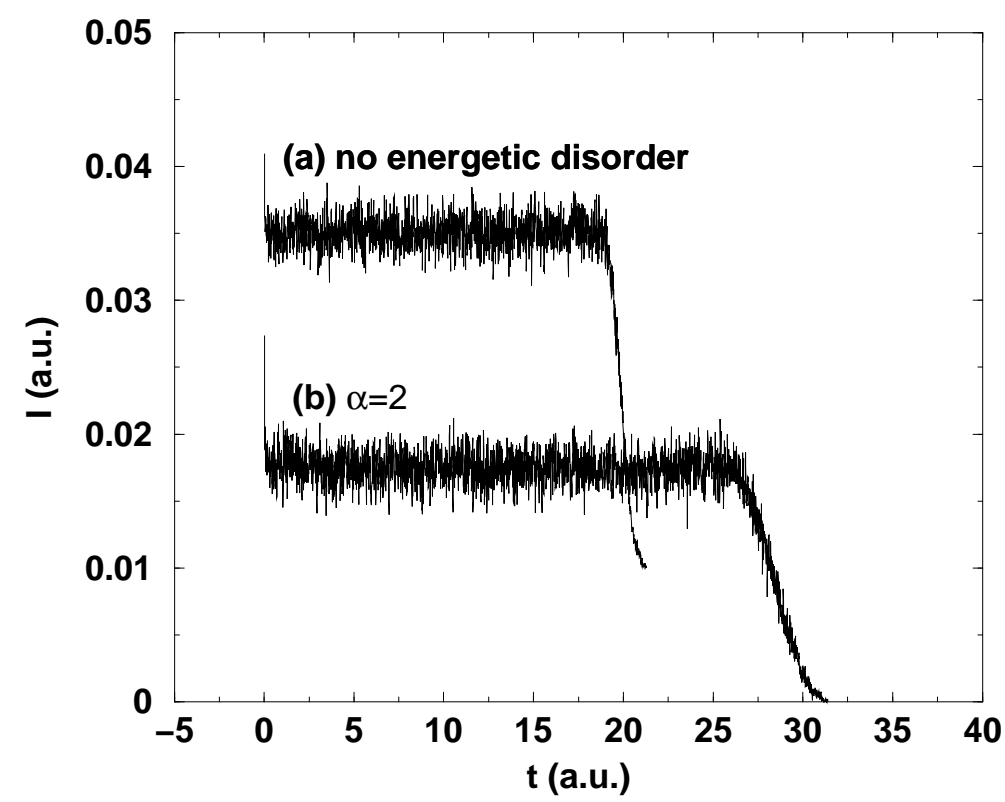

FIG. 2: Current transient for $T=300 \mathrm{~K}$ and $E=3 \times 10^{5} \mathrm{~V} / \mathrm{cm}$. Curve (a) corresponds to the case of no energetic disorder $(\delta \varepsilon=0)$, and curve (b) corresponds to $\alpha=2$. Curve (a) has been shifted along the $y$ axis for clarity. 


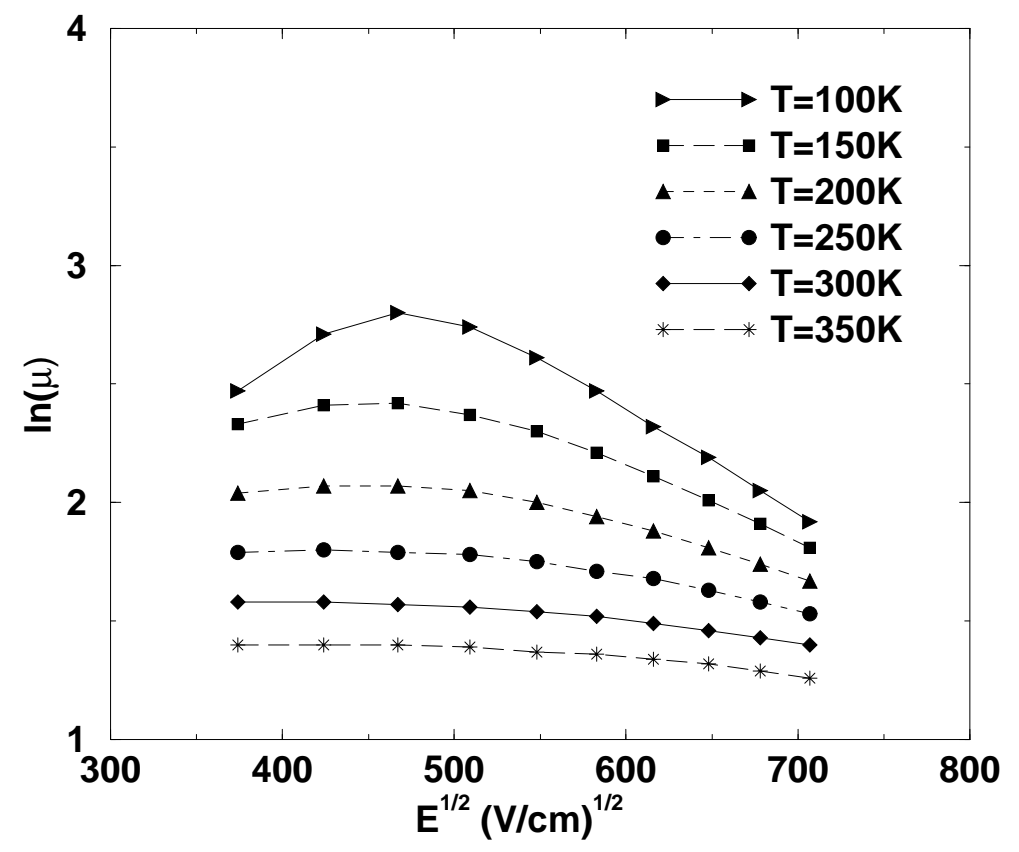

FIG. 3: The logarithm of the mobility $\ln (\mu)$ as a function of $\sqrt{E}$ and for various temperatures $T$. The energetic disorder is $\alpha=1 . \mu$ is in arbitrary units.

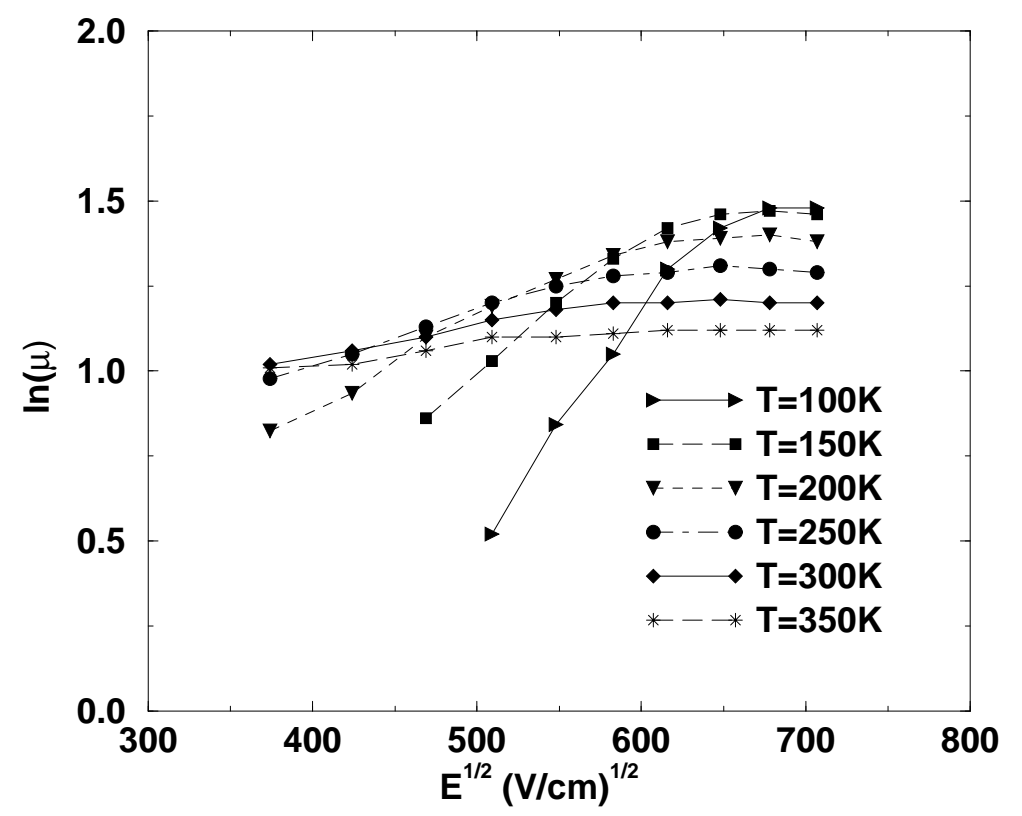

FIG. 4: The logarithm of the mobility $\ln (\mu)$ as a function of $\sqrt{E}$ and for various temperatures $T$. The energetic disorder is $\alpha=2 . \mu$ is in arbitrary units. 


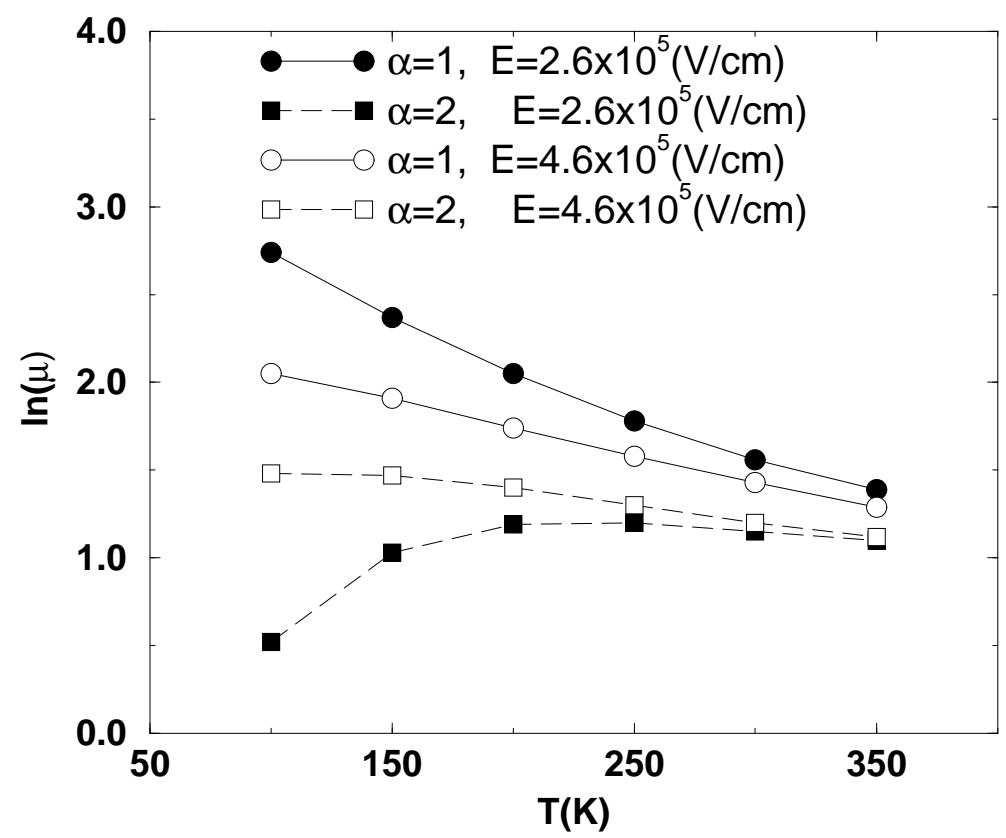

FIG. 5: The logarithm of the mobility $\ln (\mu)$ as a function of $T$. The circles correspond to $\alpha=1$, and the squares to $\alpha=2$. The filled symbols are for $E=2.6 \times 10^{5} \mathrm{~V} / \mathrm{cm}$, and the open symbols are for $E=4.6 \times 10^{5} \mathrm{~V} / \mathrm{cm}$. $\mu$ is in arbitrary units.

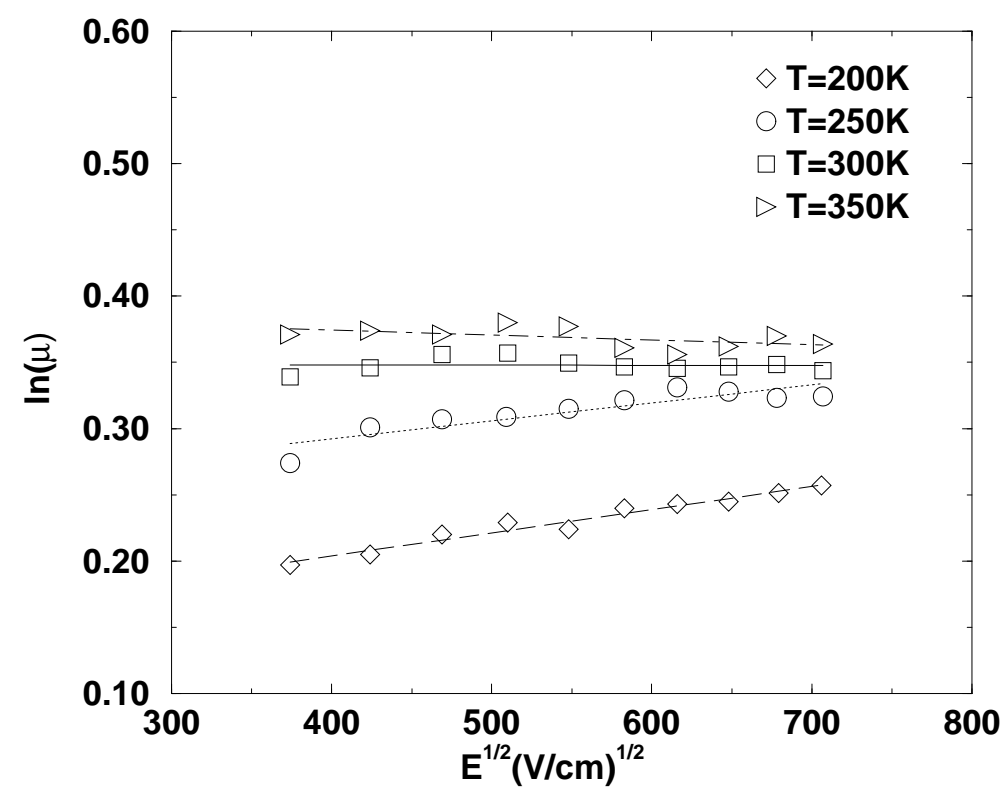

FIG. 6: The logarithm of the mobility $\mu$ as a function of $\sqrt{E}$ and for various temperatures $T$ for irregularly spaced polymer chains. The lines are a guide to the eye. The energetic disorder is equal to $\alpha=2$. $\mu$ is in arbitrary units. 


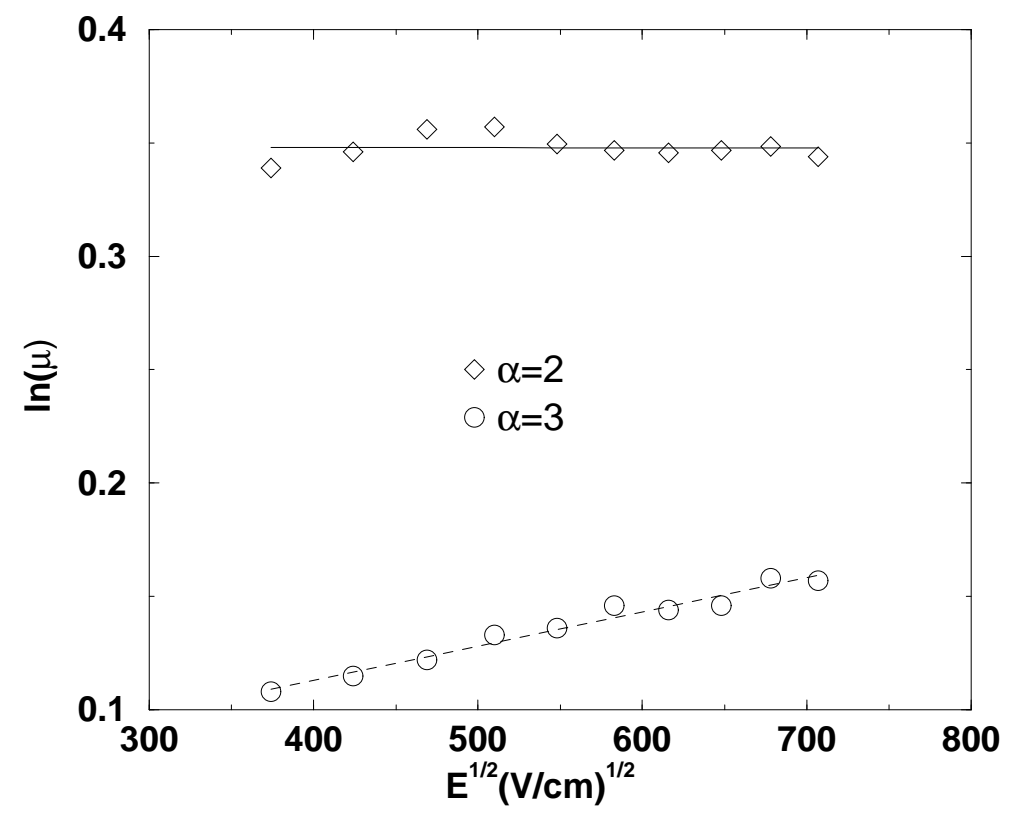

FIG. 7: The logarithm of the mobility $\mu$ as a function of $\sqrt{E}$ for irregularly spaced polymer chains, for $T=300 \mathrm{~K}$. The diamonds correspond to $\alpha=2$, and the circles to $\alpha=3$. The lines are a guide to the eye. $\mu$ is in arbitrary units.

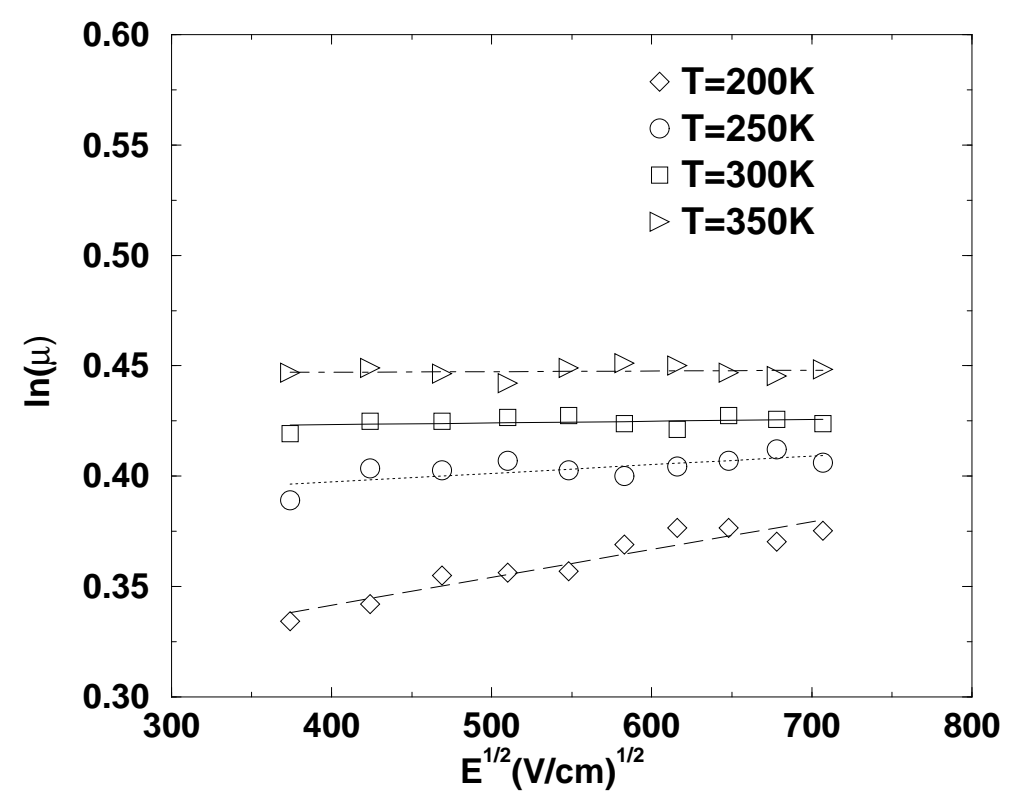

FIG. 8: The logarithm of the mobility $\mu$ as a function of $\sqrt{E}$ and for various temperatures $T$, for randomly oriented and irregularly spaced polymer chains. The energetic disorder is equal to $\alpha=2$. The lines are a guide to the eye. $\mu$ is in arbitrary units. 